\title{
Monotone Crossing Number
}

\author{
János Pach^ and Géza Tóth \\ Rényi Institute, Budapest
}

\begin{abstract}
The monotone crossing number of $G$ is defined as the smallest number of crossing points in a drawing of $G$ in the plane, where every edge is represented by an $x$-monotone curve, that is, by a connected continuous arc with the property that every vertical line intersects it in at most one point. It is shown that this parameter can be strictly larger than the classical crossing number $\mathrm{CR}(G)$, but it is bounded from above by $2 \mathrm{CR}^{2}(G)$. This is in sharp contrast with the behavior of the rectilinear crossing number, which cannot be bounded from above by any function of $\mathrm{CR}(G)$.
\end{abstract}

Keywords: crossing number, monotone drawing.

\section{Introduction}

Let $G=(V(G), E(G))$ be a graph with no loops and multiple edges, and let $V(G)$ and $E(G)$ denote its vertex set and edge set. A drawing of $G$ is an embedding of $G$ in the plane, where each vertex $v \in V(G)$ is mapped to a point and each edge $u v \in E(G)$ is mapped into a simple continuous arc connecting the images of its endpoints, but not passing through the image of any other vertex of $G$. The arcs representing the edges of $G$ are allowed to cross, but we assume for simplicity that any two arcs have finitely many points in common and no three arcs pass through the same point. A common interior point $p$ of two arcs is said to be a crossing if in a small neighborhood of $p$ one arc passes through one side of the other arc to the other side. If it leads to no confusion, the vertices and their images, as well as the edges and the arcs representing them, will be denoted by the same symbols.

In the special case where $G$ is a complete bipartite graph, the problem of minimizing the number of crossings in a drawing of $G$ was first studied by Turán [17. The question became known as the brick factory problem. It was generalized to all graphs by Erdös and Guy [3]. In two previous papers [10], 11], the authors of the present note pointed out some inconsistencies between various definitions of crossing numbers implicitly used in early publications on the subject. To distinguish between these notions, they introduced some new terminology and

\footnotetext{
* J. Pach is supported by NSF Grant CCF-08-32072, OTKA, Swiss National Science Foundation Grant 200021-125287/1, and EUROGIGA project GraDR 10EuroGIGA-OP-003. G. Tóth is supported by OTKA-K-60427, OTKA-K-75016, Bernoulli Center at EPFL, and EUROGIGA project GraDR 10-EuroGIGA-OP-003.
} 
notation. The crossing number of $G$, denoted by $\mathrm{CR}(G)$, is the smallest number of crossings in a drawing of $G$ in the plane. The pairwise crossing number, PAIR-CR $(G)$, is the smallest number of crossing pairs of edges in a drawing of $G$. If two edges cross several times, they still count as a single crossing pair, so that we have PAIR-CR $(G) \leq \mathrm{CR}(G)$ for every graph $G$. It is one of the most tantalizing open problems in this area to decide whether these two parameters coincide or at least $\mathrm{CR}(G)=O(\operatorname{PAIR}-\mathrm{CR}(G))$ holds for all graphs $G$. It was shown in 10 that $\mathrm{CR}(G)=O\left(\right.$ PAIR-CR $\left.^{2}(G)\right)$, which was successively improved in [19], 15], and [16] to $\mathrm{CR}(G)=O\left(\operatorname{PAIR}-\mathrm{CR}^{7 / 4}(G) / \log ^{3 / 2} \operatorname{PAIR}-\mathrm{CR}(G)\right)$. It is not easy to make any conjecture in this respect or even to experiment with concrete graphs. The computation of $\mathrm{CR}(G)$ and PAIR-CR $(G)$ are both NP-hard problems [7, 6], 10].

On the other hand, there is another natural parameter that can be much larger than the above two crossing numbers. LIN-CR $(G)$, the rectilinear crossing number of $G$, is the smallest number of crossings in a rectilinear drawing of $G$, that is, in a drawing where every edge is represented by a straight-line segment. We have $\mathrm{CR}(G) \leq \operatorname{LIN-CR}(G)$. Bienstock and Dean [1] constructed a series of graphs with crossing number 4 , whose rectilinear crossing numbers are arbitrarily large.

An $x$-monotone curve is a connected, continuous arc with the property that every straight-line parallel to the $y$-axis intersects it in at most one point. A drawing of $G$ is called $x$-monotone (or monotone, for short) if every edge of $G$ is represented by an $x$-monotone curve. We define $\operatorname{MON}-\mathrm{CR}(G)$, the monotone crossing number of $G$, as the smallest number of crossings in a monotone drawing of $G$. Obviously, every rectilinear drawing of $G$, in which no two vertices share the same $x$-coordinate, is a monotone drawing. Therefore, we have

$$
\mathrm{CR}(G) \leq \mathrm{MON}-\mathrm{CR}(G) \leq \mathrm{LIN}-\mathrm{CR}(G)
$$

for every graph $G$.

Monotone drawings and rectilinear drawings share many interesting properties. In particular, it was shown in 12 that every crossing-free monotone drawing of a (planar) graph $G$ can be "stretched" without changing the $x$-coordinates of the vertices. In other words, there is a crossing-free rectilinear drawing of $G$, isomorphic to the original one, in which the vertices have the same $x$-coordinates. Another example, for drawings with many crossings, is related to Conway's famous thrackle conjecture [20], which says that if a graph can be drawn in the plane such that any two edges have exactly one common points (either a common endpoint, or a crossing) then the number of edges cannot exceed the number of vertices. (The conjecture has been verified for monotone drawings [9].) In sharp contrast to these analogies, there are no graphs with bounded crossing numbers that have arbitrarily large monotone crossing numbers. In the present note, we answer a question of Fulek, Pelsmajer, Schaefer, and Štefankovič [5] by establishing the following results.

Theorem 1. Every graph $G$ satisfies the inequality

$$
\operatorname{MON}-\mathrm{CR}(G)<2 \mathrm{CR}^{2}(G) \text {. }
$$


Theorem 2. There are infinitely many graphs $G$ with arbitrarily large crossing numbers such that

$$
\operatorname{MON}-\mathrm{CR}(G) \geq \frac{7}{6} \mathrm{CR}(G)-6 \text {. }
$$

The proof of Theorem 1 is algorithmic. It is based on a recursive procedure to redraw a plane graph without changing its combinatorial structure so that in the resulting drawing any pair of vertices of the same cell can be connected by an $x$-monotone curve. See Theorem 2.2. One of the key ideas of the construction proving Theorem 2, the use of "weighted" edges or repeated paths, goes back to the paper of Bienstock and Dean [1] mentioned above. This idea was further developed and applied to related problems by Pelsmajer, Schaefer, and Stefankovič 13 and by Tóth [15].

\section{Proof of Theorem 1}

Two crossing-free (plane) drawings of a planar graph are said to be isomorphic if there is a homeomorphism of the plane which maps one to the other. In particular, it takes the unbounded cell of the first drawing to the unbounded cell of the second.

Definition 2.1. Let $\mathcal{D}$ be a crossing-free drawing of a planar graph $G$, and let $v \in V(G)$. We say that $\mathcal{D}$ is $v$-spinal if

1. $\mathcal{D}$ is a monotone drawing;

2. $v$ is the leftmost vertex;

3. any two vertices belonging to the same (bounded or unbounded) cell $C$ can be connected by an $x$-monotone curve that lies in the interior of $C$ (with the exception of its endpoints);

4. every vertical ray starting at a boundary vertex of the unbounded cell $C_{0}$ and pointing downwards lies in the interior of $C_{0}$ (with the exception of its endpoint).

Theorem 1 is an easy corollary of the following result.

Theorem 2.2. For any crossing-free drawing $\mathcal{D}$ of a planar graph and for any vertex $v$ of the unbounded cell, there is a v-spinal drawing isomorphic to $\mathcal{D}$.

It follows from the result of 12 mentioned in the introduction that every $v$ spinal drawing can be "stretched" without changing the $x$-coordinates of the vertices. That is, we can assume without loss of generality that the drawing whose existence is guaranteed by Theorem 2.2 is rectilinear. However, in the recursive argument proving Theorem 2.2 , we will not need this fact. It will be sufficient to assume that the edges are represented by $x$-monotone polygonal paths, so that in a small neighborhood of their endpoints it will make sense to talk about the slopes of these paths. 


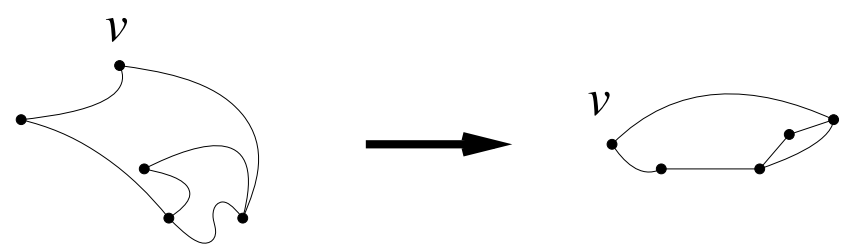

Fig. 1. A plane drawing and a $v$-spinal drawing

Before turning to the proof of Theorem 2.2, we show how Theorem 2.2 implies Theorem 1.

Proof of Theorem 1 (using Theorem 2.2). Let $G$ be any graph, and let $\mathcal{D}$ be a drawing of $G$ with $\mathrm{CR}(G)$ crossings. Let $G^{\prime} \subseteq G$ denote the subgraph consisting of all vertices of $G$ and all edges not crossed by any other edge in this drawing. Clearly, $G^{\prime}$ is a planar graph. Let $\mathcal{D}^{\prime}$ stand for the corresponding crossing-free subdrawing of $\mathcal{D}$.

Let $v$ be a vertex of the unbounded cell. By Theorem 2.2, there is a $v$-spinal drawing $\mathcal{D}^{\prime \prime}$ of $G^{\prime}$, isomorphic to $\mathcal{D}^{\prime}$. Consider now an edge $v_{1} v_{2} \in E(G) \backslash$ $E\left(G^{\prime}\right)$. In $\mathcal{D}$, this edge was represented by a curve that, with the exception of its endpoints, lied in the interior of a single cell $C^{\prime}$ in the subdrawing $\mathcal{D}^{\prime}$. Let $C^{\prime \prime}$ denote the cell in $\mathcal{D}^{\prime \prime}$, which corresponds to $C^{\prime}$. In view of condition 3 in Definition 2.1, the points representing $v_{1}$ and $v_{2}$ can be connected by an $x$ monotone curve within the cell $C^{\prime \prime}$. Let us choose such an $x$-monotone connecting curve for each edge in $E(G) \backslash E\left(G^{\prime}\right)$, so that the total number of crossings between them is as small as possible. Observe that any two such curves can cross at most once, otherwise by swapping their sections between two consecutive crossing points and slightly separating them, we could reduce the total number of crossings by 2 . During this transformation, both curves remain $x$-monotone.

Therefore, in the resulting $x$-monotone drawing of $G$, the total number of crossings is at most $\left(\begin{array}{c}|E(G)|-\left|E\left(G^{\prime}\right)\right| \\ 2\end{array}\right)$. This yields that

$$
\operatorname{MON}-\mathrm{CR}(G) \leq\left(\begin{array}{c}
|E(G)|-\left|E\left(G^{\prime}\right)\right| \\
2
\end{array}\right) .
$$

On the other hand, taking into account that every edge in $E(G) \backslash E\left(G^{\prime}\right)$ participates in at least one crossing in $\mathcal{D}$, we have

$$
|E(G)|-\left|E\left(G^{\prime}\right)\right| \leq 2 \mathrm{CR}(G) .
$$

Comparing the last two inequalities, the theorem follows.

Proof of Theorem 2.2. We proceed by induction on the number of vertices of $\mathcal{D}$. The theorem is obviously true for graphs with one or two vertices. Suppose now that $\mathcal{D}$ has $n$ vertices and that the theorem has already been proved for all drawings of graphs with fewer than $n$ vertices. Let $v$ be a vertex of the unbounded cell in $\mathcal{D}$. 
Case 1: $\mathcal{D}$ is not connected. Suppose for simplicity that it has two connected components, $\mathcal{D}_{1}$ and $\mathcal{D}_{2}$; the other cases can be treated analogously. Assume without loss of generality that $v \in \mathcal{D}_{1}$.

Subcase 1.1: $\mathcal{D}_{2}$ has a vertex $v^{\prime}$ that belongs to the unbounded cell in $\mathcal{D}$. Take a $v$-spinal drawing isomorphic to $\mathcal{D}_{1}$, and place a $v^{\prime}$-spinal drawing isomorphic $\mathcal{D}_{2}$ completely to the right of it, so that every vertex of the latter has a larger $x$-coordinate than any vertex of the former. The resulting drawing meets the requirements.

Subcase 1.2: $\mathcal{D}_{2}$ does not have a vertex that belongs to the boundary of the unbounded cell in $\mathcal{D}$. Let $C$ denote the cell in $\mathcal{D}_{1}$ that contains $\mathcal{D}_{2}$, and fix a vertex $w$ of $C$. Let $v^{\prime}$ be a vertex of the unbounded cell in $\mathcal{D}_{2}$. Take a $v$ spinal drawing isomorphic to $\mathcal{D}_{1}$, and place a very small copy of a $v^{\prime}$-spinal drawing isomorphic to $\mathcal{D}_{2}$ in the cell $C^{\prime}$ of $\mathcal{D}_{1}$ that corresponds to $C$, in a small neighborhood of the vertex that corresponds to $w$.

The resulting drawing $\mathcal{D}$ obviously satisfies conditions 1,2 , and 4 in Definition 2.1. As for condition 3 , we have to verify only that any two vertices, $v_{1}$ and $v_{2}$, that belong to the union of the boundary of $C^{\prime}$ and the outer boundary of the small $v^{\prime}$-spinal drawing isomorphic to $\mathcal{D}_{2}$ can be connected by an $x$-monotone curve that does not cross $\mathcal{D}$. This readily follows by the induction hypothesis, unless $v_{1}$ belongs to the boundary of $C^{\prime}$ and $v_{2}$ belongs to the outer boundary of the small drawing isomorphic to $\mathcal{D}_{2}$. In the latter case, move slightly downward from $v_{2}$ and then closely follow the $x$-monotone curve connecting $w$ to $v_{1}$.

CASE 2: $\mathcal{D}$ has a cut vertex $v^{\prime}$. Suppose that $\mathcal{D}=\mathcal{D}_{1} \cup \mathcal{D}_{2}$, where the only point that $\mathcal{D}_{1}$ and $\mathcal{D}_{2}$ have in common is $v^{\prime}$. Assume without loss of generality that $v$ is a vertex of $\mathcal{D}_{1}$. Note that $v$ and $v^{\prime}$ may be identical.

Let $C$ denote the cell in $\mathcal{D}_{1}$ that contains $\mathcal{D}_{2}$. In particular, $v^{\prime}$ is a vertex of $C$. In $\mathcal{D}_{2}$, the vertex $v^{\prime}$ belongs to the unbounded cell.

Take a $v$-spinal drawing isomorphic to $\mathcal{D}_{1}$, and fix a very short non-vertical segment $s$, which is incident to the point $p\left(v^{\prime}\right)$ representing $v^{\prime}$ and which lies in the cell $C^{\prime}$ that corresponds to $C$. In the special case where $v^{\prime}=v$ and $C^{\prime}$ is the unbounded cell, make sure that the $x$-coordinates of the points of $s$ are larger than the $x$-coordinate of $p\left(v^{\prime}\right)$. In addition, take a very small $v^{\prime}$-spinal drawing isomorphic to $\mathcal{D}_{2}$ such that the point representing $v^{\prime}$ coincides with $p\left(v^{\prime}\right)$. Applying a suitable orientation preserving linear transformation to this second drawing, it can be achieved that it becomes very "flat" and small, and lies in a very small neighborhood of the segment $s$, within $C^{\prime}$. Putting these two drawings together, the resulting drawing meets the requirements.

Note that, if the $x$-coordinates of the points of $s$ are smaller than the $x$ coordinate of $p\left(v^{\prime}\right)$, then the above linear transformation reverses the order of the $x$-coordinates in the $v^{\prime}$-spinal drawing isomorphic to $\mathcal{D}_{2}$. In order to preserve the combinatorial structure of the cell decomposition, we have to make sure that we use a linear transformation that preserves the orientation of the plane. 

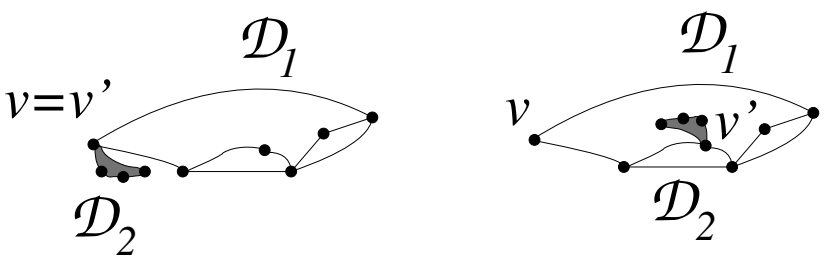

Fig. 2. Case 2. $\mathcal{D}$ has a cut vertex $v^{\prime}$

CAse 3: $\mathcal{D}$ is 2-connected. We need the following well known result.

Lemma 2.3. ([2], Proposition 3.1.2) For every 2-connected graph other than a cycle, there exists a path whose internal vertices have degree two, such that removing all edges and all internal vertices of this path, the remaining graph is still 2-connected.

Let $\mathcal{D}$ be a drawing of a cycle with vertices $v=v_{1}, v_{2}, \ldots, v_{n}$, in counterclockwise order. Then the rectilinear drawing induced by the points $p\left(v_{i}\right)=\left(i, i^{2}\right)$ is $v$ spinal and isomorphic to $\mathcal{D}$.

If $\mathcal{D}$ is not a cycle, then, according to the lemma, it can be obtained from a 2-connected drawing $\mathcal{D}_{0}$, by adding a path $P$ between two vertices, $u$ and $w$, of $\left.\mathcal{D}_{0}\right)$, which, with the exception of its endpoints, lies in the interior of a cell $C$. We distinguish two subcases.

Subcase 3.1: $v$ is a vertex of $\mathcal{D}_{0}$. Take a $v$-spinal drawing isomorphic to $\mathcal{D}_{0}$. Let $C^{\prime}$ denote the cell that corresponds to $C$ in this drawing. The vertices $u$ and $w$ belong to the boundary of this cell. Therefore, by condition 3 in Definition 2.1, $u$ and $w$ can be connected by an $x$-monotone curve within $C^{\prime}$. Put all internal vertices of $P$ along this curve, very close to $u$. The resulting drawing meets the requirements.

Subcase 3.2: $v$ is an internal vertex of $P$. Since $v$ is a vertex of the unbounded cell in $\mathcal{D}$, the cell $C$ in $\mathcal{D}_{0}$ that contains $P$, must be the unbounded cell.

Let $P=u u_{1} \cdots u_{m} v w_{1} w_{2} \cdots w_{k} w$. Assume without loss of generality that in $\mathcal{D}$ the unbounded cell lies on the left-hand side of $P$, as we traverse it from $u$ to $w$. Take a $u$-spinal drawing $\mathcal{D}_{1}$ isomorphic to $\mathcal{D}_{0}$. Place $v$ to the left and $w_{1}$ to the right of all vertices of $\mathcal{D}_{0}$.

Connect $u$ and $v$ by an $x$-monotone curve in $\mathcal{D}_{1}$, and place the vertices $u_{1}, \ldots, u_{m}$ on this curve, in this order. Then connect $v$ to $w_{1}$ by an $x$-monotone curve running above all previously drawn vertices and edges. Finally, connect $w_{1}$ to $w$ by an $x$-monotone curve which does not cross any previously drawn edges, and place the vertices $w_{2}, \ldots, w_{k}$ on this curve, in this order, very close to $w_{1}$. Adding these three curves that represent $P$ to $\mathcal{D}_{1}$, we obtain a $v$-spinal drawing isomorphic to $\mathcal{D}$, as required. 

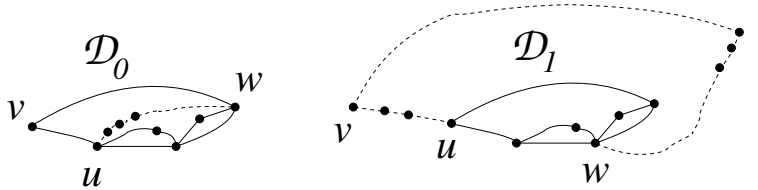

Fig. 3. Case 3. $\mathcal{D}$ is two-connected

\section{Proof of Theorem 2}

Throughout this section, let $k$ be a fixed positive integer. We construct a graph $G_{k}$ with $\mathrm{CR}\left(G_{k}\right)=6 k+6$ and MON-CR $(G)=7 k+6$, as follows.

First, we define an auxiliary graph on the vertex set $V(H)=\left\{u, w, v_{1}, \ldots, v_{9}\right\}$ such that each of its edges is red, blue, or black. Let $w$ be connected to every element of $v_{1}, \ldots, v_{9}$ by a red edge. Let $v_{1}, \ldots, v_{9}$ form a red cycle, in this order. Finally, let $H$ have three blue edges, $u v_{2}, u v_{5}$, and $u v_{8}$, and three black edges, $v_{1} v_{6}, v_{7} v_{3}, v_{4} v_{9}$. See Figure 4 . Let $H^{\prime}$ be a colored graph isomorphic to $H$ with $V\left(H^{\prime}\right)=\left\{u^{\prime}, w^{\prime}, v_{1}^{\prime}, \ldots, v_{9}^{\prime}\right\}$ and $V\left(H^{\prime}\right) \cap V(H)=\emptyset$.

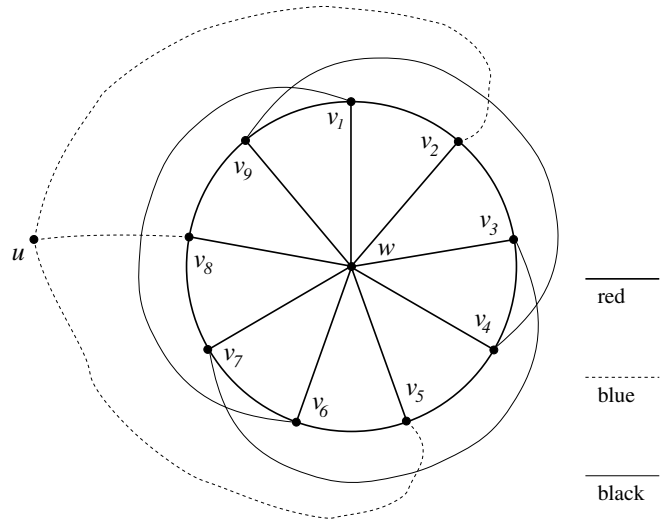

Fig. 4. Graph $H$

Let $H_{k}$ denote the graph obtained from $H$ by substituting each of its red edges by $10 k$ paths of length two and each of its blue edges by $k$ paths of length two such that the middle vertices of these paths are disjoint from one another and from all previously listed vertices. We will refer to these paths as red paths and blue paths, respectively. Let $H_{k}^{\prime}$ denote the graph with $V\left(H_{k}^{\prime}\right) \cap V\left(H_{k}\right)=\emptyset$ which can be obtained from $H^{\prime}$ in exactly the same way as $H_{k}$ was constructed from $H$.

Finally, connect $u$ to $u^{\prime}$ by a red edge, and replace this edge by $10 k$ vertex disjoint red paths of length two, as above. Denote the resulting graph by $G_{k}$. 
We start with the following simple observation.

Claim 3.1. $\mathrm{CR}\left(G_{k}\right) \leq 6 k+6$ and $\mathrm{MON}-\mathrm{CR}\left(G_{k}\right) \leq 7 k+6$.

Proof. A drawing of $G_{k}$ with $6 k+6$ crossings and a monotone drawing with $7 k+6$ crossings are depicted on Figure 5 , and Figure 6 , respectively. The thick edges and the dotted edges represent bundles consisting of $10 k$ red paths and $k$ blue paths, respectively. The paths representing the same colored edge run very close to one another and do not cross. The only difference between the two drawings is that in the first one $v_{4} v_{9}$ crosses $u v_{2}$, while in the second it crosses $u v_{5}$ and $u v_{8}$.

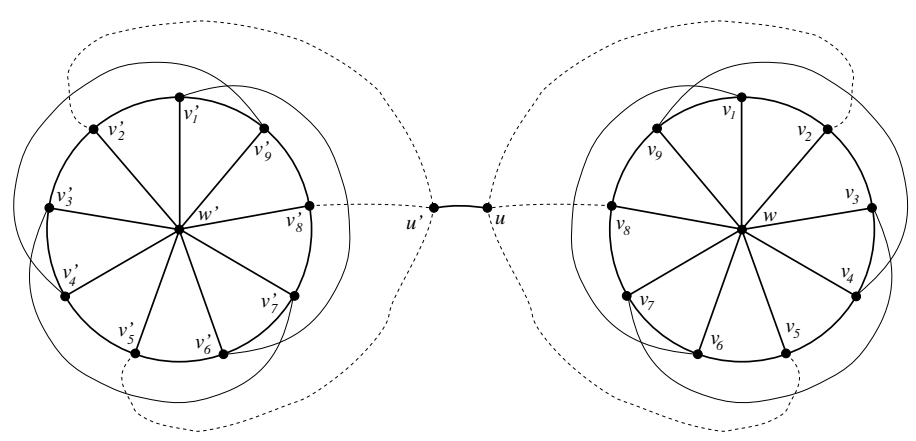

Fig. 5. A CR-optimal drawing of $G$
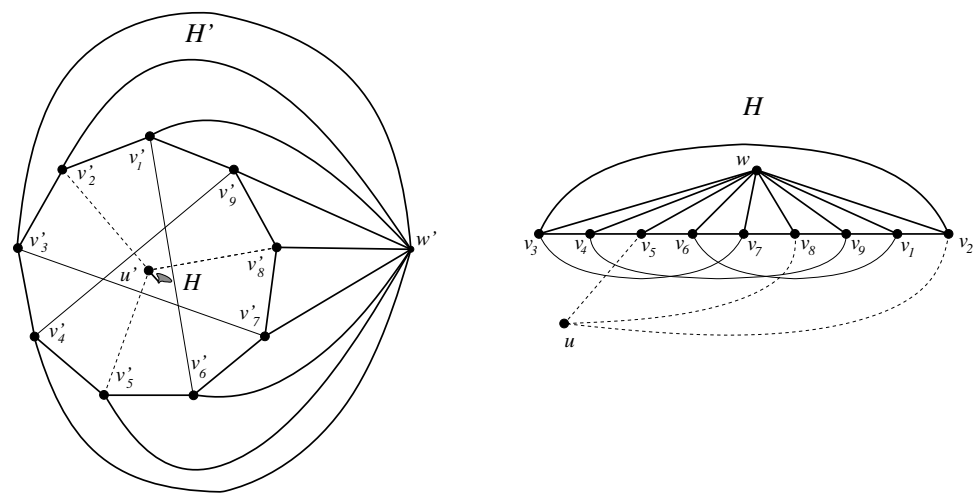

Fig. 6. Left: a MON-CR-optimal drawing of $G$. Right: the drawing of $H$, from the left.

A drawing of a graph $G$ is called CR-optimal if the number of crossings in it is $\mathrm{CR}(G)$. Analogously, a MON-CR-optimal drawing is a monotone drawing in which the number of crossings is $\operatorname{MON}-\mathrm{CR}(G)$. 
Claim 3.2. Each of the graphs $G_{k}, H_{k}$, and $H_{k}^{\prime}$ has a CR-optimal drawing and a MON-CR-optimal drawing satisfying the the following conditions. (i) The red paths substituting the same red edge run very close to one another and do not cross any edge. (ii) The blue paths substituting the same blue edge run very close to one another, do not cross one another, and cross exactly the same edges.

Proof. Let $G$ stand for one of the graphs $G_{k}, H_{k}$, or $H_{k}^{\prime}$. Let $P_{1}, \ldots, P_{m}(m=$ $10 k$ or $k$ ) denote the paths substituting the same red or blue edge. Consider a CRoptimal or a MON-CR-optimal drawing of $G$. Suppose without loss of generality that among all $P_{i}$ s the path $P_{1}$ participates in the smallest number of crossings. Redraw $P_{2}, \ldots, P_{m}$ so that they run "parallel" to $P_{1}$ and very close to it. Clearly, this transformation does not increase the total number of crossings, so that the resulting drawing remains optimal.

Suppose that $P_{1}, \ldots, P_{m}(m=10 k)$ are red paths that substitute the same red edge and run parallel to one another. If any of them crosses an edge, then all of them do. This alone creates a total of at least $10 k$ crossings, which contradicts the assumption the drawing was optimal.

Claim 3.3. $\mathrm{CR}\left(H_{k}\right)=\operatorname{MON}-\mathrm{CR}\left(H_{k}\right)=3 k+3$. Consequently, we have $\mathrm{CR}\left(G_{k}\right)=$ $6 k+6$.

Proof. The right part of Figure 6 shows a monotone drawing of $H$. From this one can easily construct a monotone drawing of $H_{k}^{\prime}$ with $3 k+3$ crossings. Therefore, we have $\mathrm{CR}\left(H_{k}\right) \leq \operatorname{MON}-\mathrm{CR}\left(H_{k}\right)=\operatorname{MON}-\mathrm{CR}\left(H_{k}^{\prime}\right) \leq 3 k+3$. As before, the thick and the dotted edges represent bundles of $10 k$ parallel red paths and bundles of $k$ parallel blue paths.

Consider a CR-optimal drawing of $H_{k}$ which satisfies the conditions in Claim 3.2. Replace now the red paths substituting the same red edge by a single red edge running along any one of those paths. The red cycle $C=v_{1} v_{2} \cdots v_{9}$ divides the rest of the plane into a bounded and an unbounded region. All points that belong to the bounded (unbounded) region are said to be inside (outside) of $C$. Assume without loss of generality that the vertex $w$ lies inside of $C$. Since no red edge is allowed to cross any other edge, the edges $v_{3} v_{7}, v_{1} v_{6}$, and $v_{4} v_{9}$, as well as the vertex $u$ with all edges incident to it, must lie outside of $C$. Thus, the edges $v_{3} v_{7}, v_{1} v_{6}$, and $v_{4} v_{9}$ are pairwise crossing. Moreover, the path $v_{2} u v_{5}$ must cross the edges $v_{3} v_{7}$ and $v_{4} v_{9}$, and the path $v_{2} u v_{8}$ must cross the edge $v_{1} v_{6}$. This already guarantees the existence of $3 k+3$ crossings, so that we have $\mathrm{CR}\left(H_{k}\right)=\operatorname{MON}-\mathrm{CR}\left(H_{k}\right)=3 k+3$.

To complete the proof of Theorem 2 , it remains to verify the following.

Claim 3.4. $\mathrm{MON}-\mathrm{CR}\left(G_{k}\right) \geq 7 k+6$.

Proof. Fix a MON-CR-optimal drawing of $G_{k}$, satisfying the conditions in Claim 3.2. As in the proof of Claim 3.3, replace every bundle of red paths substituting the same red edge by a single red edge. Let $C$ and $C^{\prime}$ denote the red cycles induced by the vertices $v_{1}, v_{2}, \ldots, v_{9}$ and $v_{1}^{\prime}, v_{2}^{\prime}, \ldots, v_{9}^{\prime}$. Both of them divide the plane into a bounded and an unbounded region, so that it makes sense to say that a point is inside or outside of $C$ or $C^{\prime}$. 
By Claim 3.2, in the original drawing of $G_{k}$, the red edges cannot cross any other edge. Suppose that a blue edge belonging to $H_{k} \subset G_{k}$ crosses an edge belonging to $H_{k}^{\prime} \subset G_{k}$. Then the number of crossings is at least $k+\operatorname{MON}-\mathrm{CR}\left(H_{k}\right)+$ MON-CR $\left(H_{k}^{\prime}\right)=7 k+6$, and we are done. Thus, we can assume that in the drawing of $G_{k}$, the blue edges of $H_{k}$ do not cross any edge of $H_{k}^{\prime}$, and analogously, the blue edges of $H_{k}^{\prime}$ do not cross any edge of $H_{k}$.

Let $v$ be the vertex of $G_{k}$ with the smallest $x$-coordinate, and suppose without loss of generality that $v \in V\left(H_{k}^{\prime}\right)$. Consider now separately the drawing of $H_{k}$ and the induced cell decomposition. By definition, $v$ lies in the unbounded cell. Observe, that if we remove edges $v_{1}^{\prime} v_{6}^{\prime}, v_{7}^{\prime} v_{3}^{\prime}, v_{4}^{\prime} v_{9}^{\prime}$ from $H_{k}^{\prime}$, that is, if we keep only the red and blue edges, we still have a connected graph. The red and blue edges of $H_{k}^{\prime}$ cannot cross any edge of $H_{k}$. Hence, all vertices of $H_{k}^{\prime}$ must lie in the unbounded cell of the cell decomposition induced by $H_{k}$.

The vertices $u$ and $u^{\prime}$ are connected by a red edge in $G_{k}$. Hence, $u$ must lie on the boundary of the unbounded cell of the cell decomposition induced by $H_{k}$. In particular, $u$ is outside of the cycle $C$. Since $w$ is connected to each edge of $C$ by a red edge, $u$ and $w$ lie on different sides of $C$. Thus, $w$ must be inside of $C$. Therefore, the edges $v_{3} v_{7}, v_{1} v_{6}, v_{4} v_{9}$, as well as the vertex $u$ together with all edges incident to it, must lie outside of $C$. Consequently, the edges $v_{3} v_{7}$, $v_{1} v_{6}, v_{4} v_{9}$ must be pairwise crossing. The edges $v_{3} v_{7}, v_{1} v_{6}, v_{4} v_{9}$ together with $C$ divide the plane into eight cells, one of which is unbounded, and $u$ must belong to this cell $\Gamma$.

Let $v_{i}$ be the vertex of $C$ with the smallest $x$-coordinate. Since $v_{3} v_{7}, v_{1} v_{6}$, $v_{4} v_{9}$ are represented by monotone curves, $v_{i}$ has to lie on the boundary of the unbounded cell $\Gamma$. We can assume without loss of generality that $1 \leq i \leq 3$. (If this is not the case, we can add 3 or 6 to all indices modulo 9.) So, $v_{i}$ is on the boundary of the unbounded cell, and $u$ is in the unbounded cell. Using the fact that the edges $v_{1} v_{2}$ and $v_{2} v_{3}$ do not cross any other edge, we can conclude that $v_{1}, v_{2}$, and $v_{3}$ all lie on the boundary of the unbounded cell $\Gamma$. See Figure 6 . Since we started with a MON-CR-optimal drawing, the edge $u v_{2}$ does not cross $v_{4} v_{9}$. The path $v_{2} u v_{5}$ crosses $v_{4} v_{9}$, so that $u v_{5}$ must cross $v_{4} v_{9}$. Analogously, $v_{2} u v_{8}$ crosses $v_{4} v_{9}$, so that $u v_{8}$ crosses $v_{4} v_{9}$. Moreover, the path $v_{2} u v_{5}$ crosses $v_{3} v_{7}$, and $v_{2} u v_{8}$ crosses $v_{1} v_{6}$. Recall from the previous paragraph that the edges $v_{3} v_{7}, v_{1} v_{6}$, and $v_{4} v_{9}$ are pairwise crossing. Summarizing, there are at least $4 k+3$ crossings between edges of $H_{k}$. By Claim 3.3, MON-CR $\left(H_{k}^{\prime}\right) \geq 3 k+3$, so that altogether $\mathrm{MON}-\mathrm{CR}\left(G_{k}\right) \geq(4 k+3)+(3 k+3) \geq 7 k+6$, as required.

\section{Concluding Remarks}

1. Another important parameter of a graph, the odd-crossing number, was introduced implicitly by Tutte [18. It is defined as the minimum number $\operatorname{ODD}-\mathrm{CR}(G)$ of all pairs of edges that cross an odd number of times, over all drawings of $G$. Clearly, for any graph $G$, we have $\operatorname{ODD}-\mathrm{CR}(G) \leq \operatorname{PAIR-CR}(G) \leq \mathrm{CR}(G) \leq$ MON-CR $(G) \leq \operatorname{LIN-CR}(G)$. Theorem 1 can be strengthened as follows. 
Corollary 4.1. Every graph $G$ satisfies the inequality

$$
\operatorname{MON}-\mathrm{CR}(G)<2 \mathrm{ODD}-\mathrm{CR}^{2}(G) .
$$

Proof. Let $\mathcal{D}$ be a drawing of $G$, in which the number of pairs of edges that cross an odd number of times is ODD-CR $(G)$. Let $G^{\prime} \subseteq G$ denote the subgraph consisting of all vertices of $G$ and all edges that do not cross any other edge an odd number of times. It was shown in [10] that $G$ has another drawing, $\mathcal{D}^{\prime}$, in which the edges belonging to $G^{\prime}$ do not participate in any crossing, and hence they form a plane graph. Every edge in $E(G) \backslash E\left(G^{\prime}\right)$ is represented by a curve that lies entirely in a cell of this plane graph. According to our Theorem 2.2, this plane graph admits a $v$-spinal (monotone) drawing for some $v \in V(G)$. By definition, we can add to this drawing all edges in $E(G) \backslash E\left(G^{\prime}\right)$, so that all of them are represented by monotone curves, and they do not cross any edge of $G^{\prime}$. Among all such monotone drawings of $G$, consider one that minimizes the total number of crossings. In this drawing, any two edges cross at most once. Thus, we have

$$
\operatorname{MON}-\mathrm{CR}(G) \leq\left(\begin{array}{c}
|E(G)|-\left|E\left(G^{\prime}\right)\right| \\
2
\end{array}\right) .
$$

On the other hand, taking into account that every edge in $E(G) \backslash E\left(G^{\prime}\right)$ participates in at least one pair of edges in $\mathcal{D}$ which cross an odd number of times, we obtain that

$$
|E(G)|-\left|E\left(G^{\prime}\right)\right| \leq 2 \mathrm{ODD}-\mathrm{CR}(G) .
$$

Comparing the last two inequalities, the corollary follows.

In 11 , we introduced the following variant of the odd-crossing number. Two edges of a graph $G$ are called independent if they do not share a vertex. Let ODD-CR $-(G)$ denote the smallest number of pairs of independent edges that cross an odd number of times, over all drawings of $G$. That is, we do not count those pairs of edges that are incident to the same vertex, even if they cross an odd number of times. Pelsmajer, Schaefer, and Štefankovič [14 managed to strengthen the result of [10, used in the proof of Corollary 4.1. They established the following result. Consider a drawing of $G$ in the plane. An edge $e \in E(G)$ is called independently even if it crosses every other edge of $G$ which is independent of $e$ an even number of times. Then $G$ has another drawing in which no independently even edge crosses any edge. Plugging this result into the above proof, we obtain the following strengthening of Corollary 4.1.

Corollary 4.1'. Every graph $G$ satisfies the inequality

$$
\mathrm{MON}-\mathrm{CR}(G) \leq 2 \mathrm{ODD}-\mathrm{CR}_{-}^{2}(G) .
$$

2. As mentioned in the Introduction, Tóth [16] proved that every graph $G$ satisfies the inequality

$$
\mathrm{CR}(G)=O\left(\operatorname{PAIR}_{-\mathrm{CR}}{ }^{7 / 4}(G) / \log ^{3 / 2} \operatorname{PAIR}-\mathrm{CR}(G)\right) .
$$


Restricting the notion of pair-crossing number to monotone drawings, we obtain another closely related graph parameter. The monotone pair-crossing number of $G$, MON-PAIR-CR $(G)$, is defined as the smallest number of crossing pairs of edges over all monotone drawings of $G$. Obviously, we have that $\operatorname{ODD}-\mathrm{CR}(G) \leq$ PAIR-CR $(G) \leq$ MON-PAIR-CR $(G)$, for any graph $G$. Valtr [19] proved that every graph $G$ satisfies the inequality MON-CR $(G)=O\left(\right.$ MON-PAIR-CR $\left.^{4 / 3}(G)\right)$.

\section{References}

1. Bienstock, D., Dean, N.: Bounds for rectilinear crossing numbers. J. Graph Theory 17, 333-348 (1993)

2. Diestel, R.: Graph Theory, 3rd edn. Graduate Texts in Mathematics, vol. 173. Springer, Berlin (2005)

3. Erdős, P., Guy, R.K.: Crossing number problems, Amer. Math. Monthly 80, 52-58 (1973)

4. Fáry, I.: On straight line representation of planar graphs. Acta Univ. Szeged. Sect. Sci. Math. 11, 229-233 (1948)

5. Fulek, R., Pelsmajer, M.J., Schaefer, M., Štefankovič, D.: Hanani-Tutte, monotone drawings, and level-planarity (to appear)

6. Garey, M.R., Johnson, D.S.: Crossing number is NP-complete, SIAM J. Alg. Disc. Meth. 4, 312-316 (1983)

7. Garey, M.R., Johnson, D.S., Stockmeyer, L.J.: Some simplified NP-complete graph problems. Theoretical Computer Science 1, 237-267 (1976)

8. Guy, R.K.: The decline and fall of Zarankiewicz's theorem. In: Guy, R.K. (ed.) Proof Techniques in Graph Theory, pp. 63-69. Academic Press, New York (1969)

9. Pach, J., Sterling, E.: Conways conjecture for monotone thrackles. Amer. Math. Monthly 118, 544-548 (2011)

10. Pach, J., Tóth, G.: Which crossing number is it, anyway? J. Combin. Theory Ser. B 80, 225-246 (2000)

11. Pach, J., Tóth, G.: Thirteen problems on crossing numbers. Geombinatorics 9, 194-207 (2000)

12. Pach, J., Tóth, G.: Monotone drawings of planar graphs. J. Graph Theory 46, 39-47 (2004)

13. Pelsmajer, M.J., Schaefer, M., Štefankovič, D.: Odd crossing number and crossing number are not the same. Discrete Comput. Geom. 39, 442-454 (2008)

14. Pelsmajer, M.J., Schaefer, M., Štefankovič, D.: Removin independently even crossings. SIAM J. Discrete Math. 24, 379-393 (2010)

15. Tóth, G.: Note on the pair-crossing number and the odd-crossing number. Discrete Comput. Geom. 39, 791-799 (2008)

16. Tóth, G.: A better bound for the pair-crossing number (manuscript)

17. Turán, P.: A note of welcome. J. Graph Theory 1, 7-9 (1977)

18. Tutte, W.T.: Toward a theory of crossing numbers. J. Combinatorial Theory 8, 45-53 (1970)

19. Valtr, P.: On the pair-crossing number. In: Combinatorial and Computational Geometry. Math. Sci. Res. Inst. Publ., vol. 52, pp. 569-575. Cambridge Univ. Press, Cambridge (2005)

20. Woodall, D.R.: Thrackles and deadlock. In: Welsh, D.J.A. (ed.) Combinatorial Mathematics and Its Applications, pp. 335-348. Academic Press (1969) 